\title{
On a boundary layer problem related to the gas flow in shales
}

\section{Citation}

Barenblatt, G. I., P. J. M. Monteiro, and C. H. Rycroft. 2013. “On a Boundary Layer Problem Related to the Gas Flow in Shales." Journal of Engineering Mathematics 84 (1) (January 16): 11 18. doi:10.1007/s10665-012-9612-7.

\section{Published Version}

doi:10.1007/s10665-012-9612-7

\section{Permanent link}

http://nrs.harvard.edu/urn-3:HUL.InstRepos:32197090

\section{Terms of Use}

This article was downloaded from Harvard University's DASH repository, and is made available under the terms and conditions applicable to Other Posted Material, as set forth at http:// nrs.harvard.edu/urn-3:HUL.InstRepos:dash.current.terms-of-use\#LAA

\section{Share Your Story}

The Harvard community has made this article openly available.

Please share how this access benefits you. Submit a story.

\section{Accessibility}




\title{
On a boundary layer problem related to the gas flow in shales
}

\author{
G. I. Barenblatt - P. J. M. Monteiro • \\ C. H. Rycroft
}

Received: 24 July 2012 / Accepted: 3 December 2012 / Published online: 16 January 2013

(C) US Government 2013

\begin{abstract}
The development of gas deposits in shales has become a significant energy resource. Despite the already active exploitation of such deposits, a mathematical model for gas flow in shales does not exist. Such a model is crucial for optimizing the technology of gas recovery. In the present article, a boundary layer problem is formulated and investigated with respect to gas recovery from porous low-permeability inclusions in shales, which are the basic source of gas. Milton Van Dyke was a great master in the field of boundary layer problems. Dedicating this work to his memory, we want to express our belief that Van Dyke's profound ideas and fundamental book Perturbation Methods in Fluid Mechanics (Parabolic Press, 1975) will live on-also in fields very far from the subjects for which they were originally invented.
\end{abstract}

Keywords Porous media $\cdot$ Subterranean fluid mechanics

\section{Introduction}

A wealth of information about the geological structure of shales covering length scales from the pore size to deposit size can be found in the article of Silin and Kneafsey [1] (see also [2]). The structure of a typical microelement can be represented as follows (Fig. 1): there exists a matrix, which is a porous and perhaps even fissurized medium. Its permeability, although sometimes small, nevertheless is sufficient for consideration by the classical approach of subterranean fluid mechanics [3-5]. Within the matrix, inclusions are distributed, having moderate, and sometimes even large, porosity, but very low permeability, on the order of a nanodarcy $\left(\sim 10^{-21} \mathrm{~m}^{2}\right)$ or less; the standard permeability of oil and gas deposits is 100 millidarcys $\left(\sim 10^{-13} \mathrm{~m}^{2}\right)$. The low permeability of the inclusions is

G. I. Barenblatt · C. H. Rycroft ( $\varangle)$

Department of Mathematics, University of California, Berkeley, CA 94720, USA

e-mail: chr@math.berkeley.edu

G. I. Barenblatt · C. H. Rycroft

Department of Mathematics, Lawrence Berkeley National Laboratory, Berkeley, CA 94720, USA

G. I. Barenblatt

Institute of Oceanology, Russian Academy of Sciences, Moscow 119997, Russia

P. J. M. Monteiro

Department of Civil and Environmental Engineering, University of California, Berkeley, CA 94720, USA 


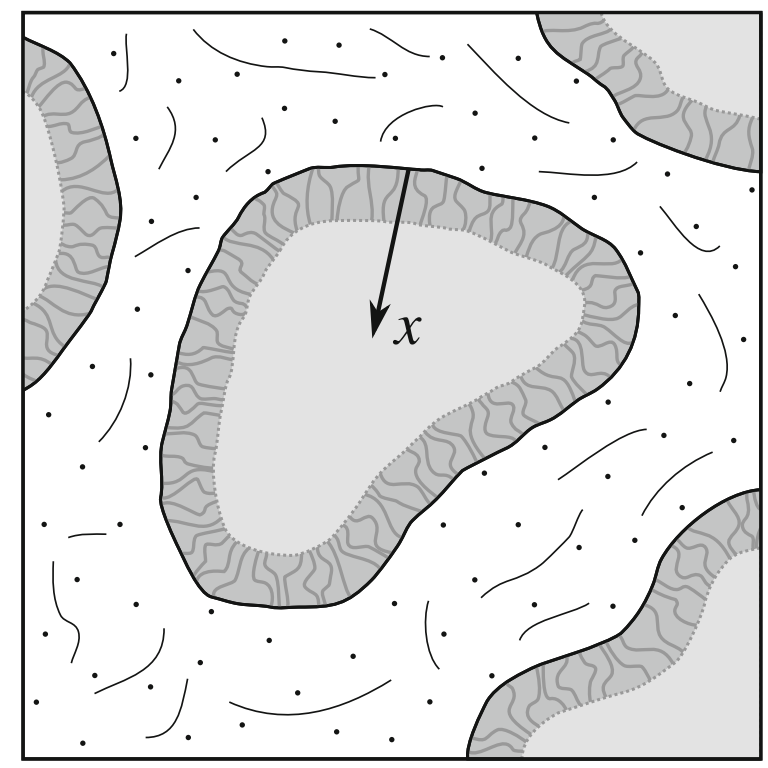

Fig. 1 A low-permeability inclusion (gray) is embedded within a porous matrix shown by the white, dotted region, which may be fissurized. When exploitation begins and the pressure in the matrix is lowered, a boundary layer of gas flow (dark gray) develops and grows in width over time. The flow in the boundary layer is determined as a function of time and the coordinate $x$, which is normal to the inclusion boundary and directed inside the inclusion

related to the smallness of pores or channels whose diameter, in contrast to ordinary rocks forming oil and gas deposits $(1-100 \mu \mathrm{m})$, is on the order of nanometers.

In typical shales, it is thought that a large majority of the gas is contained within the inclusions. Due to the extremely low permeability of the inclusions, classical subterranean fluid mechanics would predict a negligible amount of flow on a realistic time scale. However, experimental data point to substantial amounts of flow for reasons that are not fully understood. Here we develop a mathematical model of gas flow in shales to explain this observation, based upon the hypothesis that the inclusions undergo a nanostructural transformation when subjected to a very large pressure gradient, causing a substantial increase in permeability. The mathematical model makes predictions about the expected gas flow rate from a shale deposit.

\section{Mechanism of gas flow from the inclusions}

When the exploitation of a gas deposit in shales begins, the gas pressure in the matrix is reduced relatively rapidly $[3,5]$. Due to the extremely low permeability of the inclusions, there appears rather quickly a pressure difference at the boundaries between the inclusions and the matrix. The permeability of the inclusions is small, but nevertheless nonzero, and therefore in the narrow region at the boundaries between the inclusions and the matrix there appears a very large pressure gradient.

Our basic hypothesis is formulated as follows. Due to the large pressure gradient, there appears a boundary layer in the inclusions (shown in dark gray in Fig. 1) where the shale undergoes a nanostructural transformation, corresponding to breakage and/or deformation of the pores and channels in the material. This causes the boundary layer to become significantly more permeable for gas. Since the pores are narrow, the influence of the Knudsen number $\mathrm{Kn}$, defined as the ratio of the molecular mean free path to a representative length scale, should also be assumed. This leads to an expression for permeability of the form

$k=F(\nabla p, \mathrm{Kn})$. 
Two items should be mentioned. Firstly, due to the small thickness of the boundary layer, the tangential components of the pressure gradient are negligibly small, so the gas flow is one-dimensional, and in fact the permeability in the boundary layer depends on the component $\partial_{x} p$, where $x$ (Fig. 1) is the coordinate along the normal to the inclusion boundary and directed inside the inclusion. Secondly, in a more general case, the permeability could also depend on the pressure $p$, although this is not considered in detail here (dependence on pressure, but not the dependence on the pressure gradient, was used by Silin et al. [2]).

It is important that the pores in the boundary layer have no characteristic, selected length scale, and therefore in the intermediate asymptotic interval

$\lambda \ll x \ll \Lambda$

there is no characteristic quantity having the dimension of the pressure gradient. Here $\Lambda$ is the characteristic length size of the inclusion and $\lambda$ is the fundamental nanometer length scale, $1 \AA$, a pixel in the description of nanoscale phenomena [6]. From this assumption it follows that the permeability in the boundary layer is the power function of the pressure gradient

$k=A\left(\partial_{x} p\right)^{m}$,

where, in a more general case, $A$ and $m$ can depend on the Knudsen number and $A$ can also depend on the pressure. Indeed, if $\partial_{x} p$ is denoted by $z$, then the ratio of permeabilities at two different values of $z, z=z_{1}$ and $z=z_{2}$, is $F\left(z_{1}\right) / F\left(z_{2}\right)$. If we pass to different units of pressure or length, then this ratio will be equal to $F\left(\alpha z_{1}\right) / F\left(\alpha z_{2}\right)$, where $\alpha$ is a constant factor. If there exists no other characteristic argument of the dimension of pressure gradient, then the ratio of permeabilities preserves the same value, so that

$\frac{F\left(z_{1}\right)}{F\left(z_{2}\right)}=\frac{F\left(\alpha z_{1}\right)}{F\left(\alpha z_{2}\right)}$.

We have the right to select $\alpha=z_{2}^{-1}$ such that this equation assumes the form

$\frac{F\left(z_{1}\right)}{F\left(z_{2}\right)}=\frac{F\left(z_{1} / z_{2}\right)}{F(1)}$

Differentiating by $z_{1}$ and setting $z_{1}=z_{2}=z$ yields

$\frac{F^{\prime}(z)}{F(z)}=\frac{1}{z} \frac{F^{\prime}(1)}{F(1)}$,

whereupon integration gives $F(z)=A z^{m}$.

\section{Model of gas flow from the inclusions}

Using the basic equation of mass conservation

$\phi \frac{\partial \rho}{\partial t}+\nabla \cdot \mathbf{j}=0$

and the Darcy law

$\mathbf{j}=-\frac{k}{\mu} \rho \nabla p$,

where $\phi$ is the porosity and $\mu$ is the viscosity of gas, we obtain the basic equation

$\phi \frac{\partial \rho}{\partial t}=\frac{\partial}{\partial x}\left(\frac{A \rho}{\mu}\left(\partial_{x} p\right)^{m+1}\right)$.

This equation would remain valid in the more general case where $\phi, \mu$, and $A$ could have a dependence on $p$, although in the present article we do not consider this and assume that they are constants. We assume further that the gas is 
thermodynamically perfect and that the flow is isothermal, so that $\rho(p)=C p$, where $C$ is a temperature-dependent constant. Using these assumptions, we reduce Eq. (8) to the final equation

$\frac{\partial \rho}{\partial t}=\kappa \frac{\partial}{\partial x}\left(p\left(\partial_{x} p\right)^{m+1}\right)$,

where

$\kappa=\frac{A}{\phi \mu}$

is a constant that can depend on the Knudsen number. In the classical case where $m=0$ and $A=k$, the BoussinesqLeibenson equation

$\frac{\partial p}{\partial t}=\kappa \frac{\partial^{2}}{\partial x^{2}}\left(p^{2}\right)$

is obtained, where the constant this time is equal to $\kappa=k /(2 \phi \mu)$.

\section{Boundary conditions and self-similar solution to the boundary layer problem}

Equation (8) for the problem is complemented by the two boundary conditions

$p(0, t)=p_{0}, \quad p(\infty, t)=P$

and the initial condition

$p\left(x, t_{0}\right)=P$,

where $t_{0}$ is the time of the beginning of the exploitation. $P$ is the gas pressure in the pristine state, and $p_{0}$ is the pressure in the matrix, established rapidly - we can assume instantaneously — after the beginning of the exploitation, and after that remaining constant. The derivation of the second boundary condition in Eq. (12) follows the usual steps of classical boundary layer theory in a viscous fluid. It is important to note that the relative depression $\left(P-p_{0}\right) / P$ is small- this is a well-known fact in the practice of the development of gas deposits. We can now solve the mixed Cauchy-boundary value problem given by Eqs. (9), (12), and (13). The solution depends on the quantities

$x, t-t_{0} ; \kappa, P, p_{0}$.

The dimensions of these quantities in the $L T[p]$ class are

$[x]=L, \quad\left[t-t_{0}\right]=T, \quad[\kappa]=\frac{L^{m+2}}{T}[p]^{-(m+1)}, \quad\left[p_{0}\right]=[P]=[p]$.

Standard application of dimensional analysis gives

$p=P f\left(\frac{x}{\left(\kappa\left(t-t_{0}\right) P^{m+1}\right)^{1 /(m+2)}}\right)$,

so that the solution is self-similar. We denote the independent variable $\xi$ as

$\xi=\frac{x}{\left(\kappa\left(t-t_{0}\right) P^{m+1}\right)^{1 /(m+2)}}$,

so that

$p=\operatorname{Pf}(\xi)$.

Substituting Eqs. (17) and (18) into Eq. (9) with conditions given by Eqs. (13) and (12), we obtain

$\frac{1}{m+2} \xi \frac{\mathrm{d} f}{\mathrm{~d} \xi}+\frac{\mathrm{d}}{\mathrm{d} \xi}\left(f\left(\frac{\mathrm{d} f}{\mathrm{~d} \xi}\right)^{m+1}\right)=0$ 

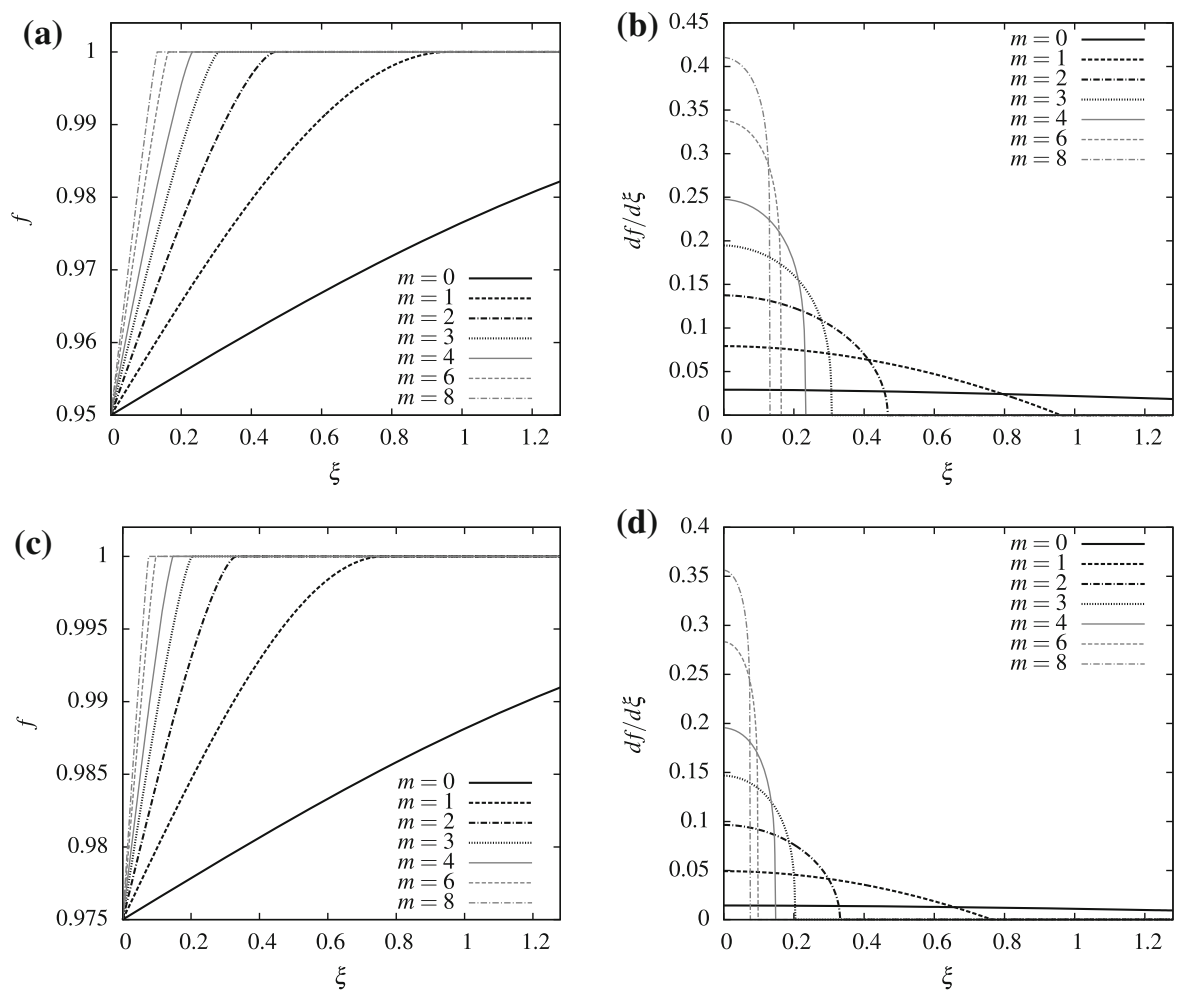

Fig. 2 Plots of $\mathbf{a} f$ and $\mathbf{b ~ d} f / \mathrm{d} \xi$ for the case of $\sigma=0.95$, and plots of $\mathbf{c} f$ and $\mathbf{d ~} \mathrm{d} f / \mathrm{d} \xi$ for the case of $\sigma=0.975$

with the boundary conditions at the different ends of the interval being

$f(0)=\frac{p_{0}}{P}=\sigma, \quad f(\infty)=1$.

Equation (19) can be solved numerically using the Runge-Kutta method. To do this, it is convenient to rewrite the equation as a first-order system

$\frac{\mathrm{d} f}{\mathrm{~d} \xi}=u$,

$(m+1) u^{m} f \frac{\mathrm{d} u}{\mathrm{~d} \xi}=-\frac{\xi u}{m+2}-u^{m+2}$.

The shooting method is used, whereby the system given by Eqs. (21) and (22) is treated as an initial-value problem. An integration step of $4 \times 10^{-6}$ is used, which is small enough to ensure numerical errors will be negligible.

We now come to the results of numerical integration. Figure $2 \mathrm{a}, \mathrm{b}$ shows graphs of $f(\xi)$ and $\mathrm{d} f / \mathrm{d} \xi$ for the case where $\sigma=0.95$ for various values of $m$. The most significant feature suggested by the computations is that for $m>0$ the solutions have a compact support: pressure values are different from 1 only over a finite interval $0 \leq \xi<\xi_{0}(m)$. Figure 2c, d shows the same quantities for the case where $\sigma=0.975$, indicating that for a given value of $m$ the corresponding value of $\xi_{0}(m)$ is reduced. In Fig. 3 the values of $\mathrm{d} f / \mathrm{d} \xi$ at $\xi=0$ that are determined using the shooting method, and the values of $\xi_{0}(m)$, are plotted as a function of $m$ for the two values of $\sigma$ that are considered.

Figure $4 \mathrm{a}$ shows plots of the pressure gradient for $\sigma=0.95$ when rescaled by the values from Fig. 3. In Fig. $4 \mathrm{~b}$, plots of rescaled permeability are given, by taking a rescaled pressure gradient to the $m$ th power. It is instructive that the result is universal ( $m$-independent); the universality was found by numerical integration. For $\sigma$ close to one, the universality can be proved analytically. Indeed, in such cases $u=\mathrm{d} f / \mathrm{d} \xi$ is small, $f$ is close to one, and 

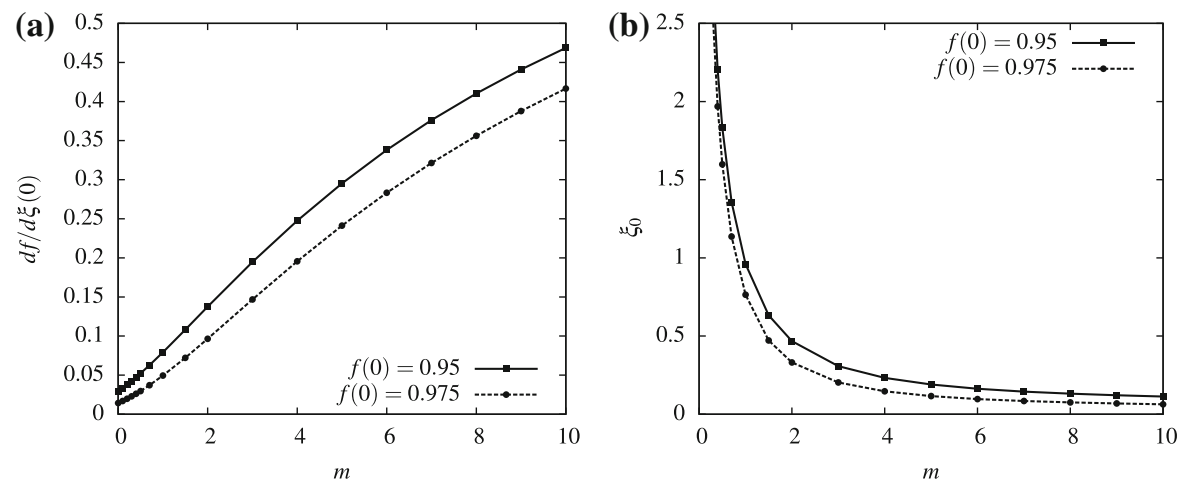

Fig. 3 Plots of values of $\mathbf{a} \mathrm{d} f / \mathrm{d} \xi$ that are found using the shooting method as a function of $m$, and $\mathbf{b} \xi_{0}$ as a function of $m$ for the two values of $\sigma$ that have been considered
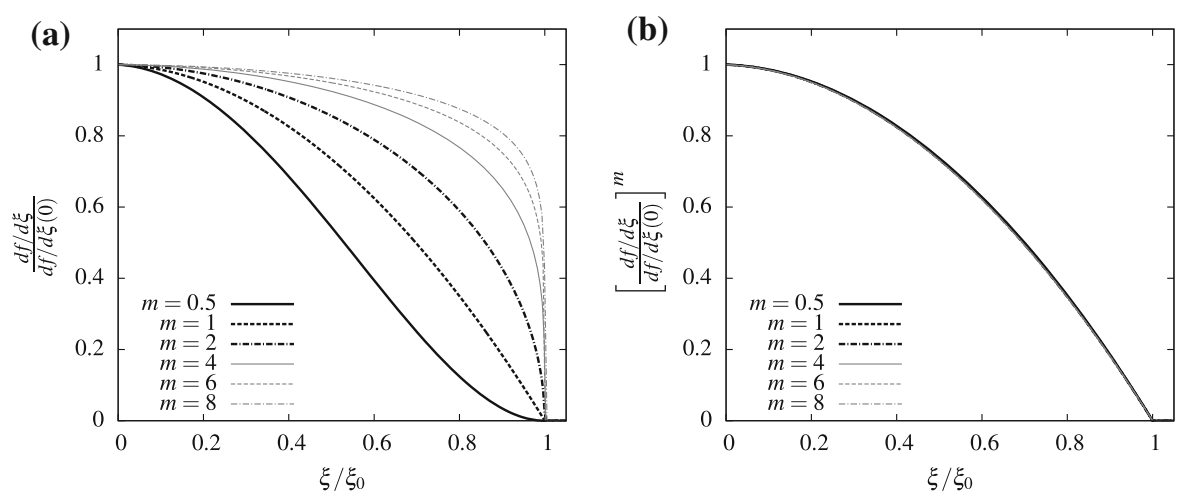

Fig. 4 Plots of $\mathbf{a ~ d ~} f / \mathrm{d} \xi$ when rescaled by values in Fig. 3 and $\mathbf{b}$ rescaled permeability, for various values of $m$, for the case where $\sigma=0.95$

$u^{m+2} \ll u$. Therefore, the third term in Eq. (22) can be neglected, and the equation takes the form

$(m+1) u^{m} \frac{\mathrm{d} u}{\mathrm{~d} \xi}+\frac{\xi u}{m+2}=0$.

Integration of this equation under the condition $u=0$ at $\xi=\xi_{0}$ gives

$u^{m}=-\frac{m \xi^{2}}{2(m+1)(m+2)}+$ const.

At $\xi=\xi_{0}, u=\mathrm{d} f / \mathrm{d} \xi=0$, so that

$u^{m}=\left(\frac{\mathrm{d} f}{\mathrm{~d} \xi}\right)^{m}=\frac{m}{2(m+1)(m+2)}\left(\xi_{0}^{2}-\xi^{2}\right)$

or

$\left(\frac{\frac{\mathrm{d} f}{\mathrm{~d} \xi}}{\left(\frac{\mathrm{d} f}{\mathrm{~d} \xi}\right)_{\xi=0}}\right)^{m}=1-\frac{\xi^{2}}{\xi_{0}^{2}}$.

Figure 5 demonstrates the smallness of the difference between the rescaled permeability obtained by numerical integration and the parabola given by Eq. (26).

The prediction of the gas flow rate for a given deposit is of crucial importance. According to the previous analysis, we obtain for the inflow rate per unit volume $Q$ at time $t$ 
Fig. 5 Plots of difference between rescaled permeability and the approximate parabolic solution, for several values of $m$, for the case where $\sigma=0.95$

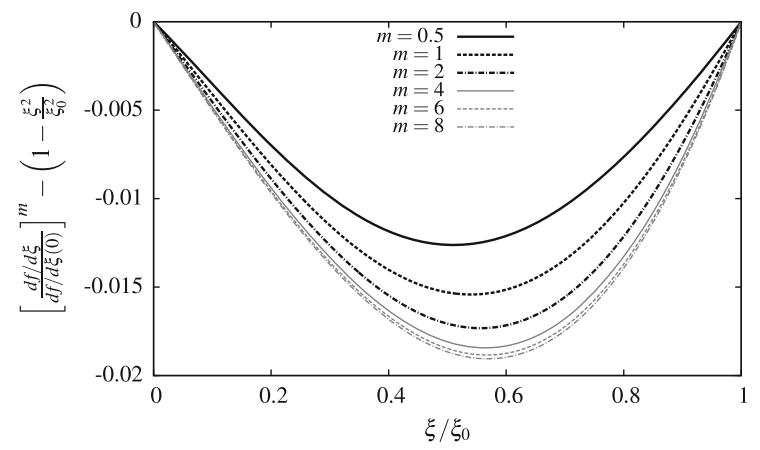

$$
\begin{aligned}
Q & =S\left(\frac{k \rho_{0}}{\mu}\left(\partial_{x} p\right)\right)_{x=0} \\
& =\frac{S A \rho_{0}}{\mu} P^{m+1}\left(\frac{\mathrm{d} f(0)}{\mathrm{d} \xi}\right)^{m+1} \frac{1}{\left[\kappa\left(t-t_{0}\right) P^{m+1}\right]^{\frac{m+1}{m+2}}} \\
& =\frac{S A \rho_{0}}{\mu} P^{\frac{m+1}{m+2}}\left(\frac{\mathrm{d} f(0)}{\mathrm{d} \xi}\right)^{m+1} \frac{1}{\left(\kappa\left(t-t_{0}\right)\right)^{\frac{m+1}{m+2}}} \\
& =\frac{S A^{\frac{1}{m+2}} \phi^{\frac{m+1}{m+2}} \rho_{0}}{\mu^{\frac{1}{m+2}}} P^{\frac{m+1}{m+2}}\left(\frac{\mathrm{d} f(0)}{\mathrm{d} \xi}\right)^{m+1} \frac{1}{\left(t-t_{0}\right)^{\frac{m+1}{m+2}}} .
\end{aligned}
$$

Here $S$ is the specific surface of the inclusions (surface per unit volume). Equation (27) clearly indicates that the inflow rate decreases over time according to a power law. The exponent in the decay rate varies from $-\frac{1}{2}$ for $m=0$ down to -1 as $m \rightarrow \infty$. Classical subterranean fluid mechanics would predict a decay of the gas inflow rate according to $\left(t-t_{0}\right)^{-1 / 2}$, and thus the model we present predicts that higher rates of decay will be observed.

\section{Conclusion}

Gas recovery from shales is considered today to be one of the most promising directions in natural gas production. In the present article a mathematical model of the gas flow in shales is presented based on the assumption of a nanostructural transformation that significantly increases the permeability of low-permeability inclusions containing the (basic) amount of gas in shales. A boundary layer on the surface of the inclusions is formed during the process of exploitation. It is demonstrated that the flow in this boundary layer is self-similar, and a complete solution is constructed. The ultimate formula for the gas inflow rate from the inclusions to the porous matrix is obtained, and it predicts a range of decay rates over time that are higher than for classical subterranean fluid mechanics.

Acknowledgments The authors express their special gratitude to Dmitriy B. Silin, whose work and presentations motivated our study. This publication was based on the work supported in part by Award No. KUS-I1-004021, made by King Abdullah University of Science and Technology. G.I.B. and C.H.R. were partially supported by the Director, Office of Science, Computational and Technology Research, U.S. Department of Energy under Contract No. DE-AC02-05CH11231.

\section{References}

1. Silin DB, Kneafsey TJ (2010) Gas shale: from nanometer-scale observations to well-modeling. Society of Petroleum Engineers, Richardson, Texas, Paper no. 149489. doi:10.2118/149489-MS

2. Silin DB, Kneafsey TJ, Ajo-Franklin JB, Nico P (2010) A multimodal 3D imaging study of natural gas flow in tight sands. Society of Petroleum Engineers, Richardson, Texas, Paper no. 146611. doi:10.2118/146611-MS

3. Streltsova TD (1988) Well testing in heterogenous media. Wiley, New York 
4. Barenblatt GI, Entov VM, Ryzhik VM (1990) Theory of fluid flows through natural rocks. Kluwer, Dordrecht

5. Shchelkachev VN (2004) Main principles of oil field development. Oil and Gas publishing house, Moscow

6. Barenblatt GI, Monteiro PJM (2010) Scaling laws in nanomechanics. Phys Mesomech 13(5-6):245-248 